\title{
Investigating Multiple Pheromones in Swarm Robots - A Case Study of Multi-Robot Deployment
}

\author{
Tian Liu ${ }^{1, \dagger}$, Xuelong Sun ${ }^{1, \dagger, *}$, Cheng $\mathrm{Hu}^{2}$, Qinbing $\mathrm{Fu}^{1,2}$, Hamid Isakhani ${ }^{1}$, and Shigang Yue ${ }^{1,2, *}$
}

\begin{abstract}
Social insects are known as the experts in handling complex task in a collective smart way although their small brains contain only limited computation resources and sensory information. It is believed that pheromones play a vital role in shaping social insects' collective behaviours. One of the key points underlying the stigmergy is the combination of different pheromones in a specific task. In the swarm intelligence field, pheromone inspired studies usually focus one single pheromone at a time, so it is not clear how effectively multiple pheromones could be employed for a collective strategy in the real physical world. In this study, we investigate multiple pheromone-based deployment strategy for swarm robots inspired by social insects. The proposed deployment strategy uses two kinds of artificial pheromones; the attractive and the repellent pheromone that enables micro robots to be distributed in desired positions with high efficiency. The strategy is assessed systematically by both simulation and real robot experiments using a novel artificial pheromone platform $\operatorname{ColCOS} \Phi$. Results from the simulation and real robot experiments both demonstrate the effectiveness of the proposed strategy and reveal the role of multiple pheromones. The feasibility of the $\operatorname{ColCOS} \Phi$ platform, and its potential for further robotic research on multiple pheromones are also verified. Our study of using different pheromones for one collective swarm robotics task may help or inspire biologists in real insects' research.
\end{abstract}

\section{INTRODUCTION}

Pheromone, a term coined by Peter Karlson and Martin Lüscher in 1959, is a kind of secreted compound that can trigger specific individual behaviours underlying some complex and flexible collective performances [1]. There are many types of pheromones in the social insects' world which are crucial for the colony's survival and reproduction [2]. For example, the sex pheromone relayed by a female can attract the male of the same species to improve the possibility of mating, the oviposition-deterring pheromone [3] will repel other females from oviposition near the site where larvae have already been present, which improves the oviposition efficiency of the whole colony. There are numerous properties that make pheromones a significant source of inspiration for solving the problems in swarm robotics [4], [5]. For example, the ant and bee colony optimisation [6], the pheromone guided robot dispersion or

This work was supported by EU Horizon 2020 project STEP2DYNA(691154) and ULTRACEPT(778062).

Joint first authors.

*Corresponding authors, (xsun,syue@lincoln.ac.uk).

${ }^{1}$ Computational Intelligence Laboratory/Lincoln Center for Autonomous Systems, School of Computer Science, University of Lincoln, LN6 7TS, United Kingdom.

${ }^{2}$ Machine Life and Intelligence Research Center, School of Mechanical and Electrical Engineering, Guangzhou University, Guangzhou 510006, China. deployment [7], [8], [9] and so on. However, most of these studies only utilise one single pheromone in their solution except for few studies using multiple pheromones but in a simulated environment [10], [11]. One pheromone for one collective task is not the case in the real animal world. For instances, in the ant's food recruitment task, at least three kinds of pheromones are involved [12], [13]. Robinson et al. [12] argued that these different pheromones working together can greatly improve the food recruitment efficiency and save the colony energy. Thus, from the bio-inspired perspective, we want to ask what is the role of multiple pheromones in solving swarm robotic problems? As one of the fundamental and challenging tasks for swarm robotics is to deploy robots in an environment to perform surveillance, collect data, or establish a sensor network [14], in this paper, we take the deployment problem as an entry to investigate the multiple pheromones focused scenarios.

As compared to a single pheromone study, multiple pheromones are not as much investigated and to compensate for this, here we devise a multi-agents deployment strategy based on multiple pheromones communication. Inspired by animals, this strategy applies attractive pheromone to guide the agents to the goal positions and repellent pheromone to tell the agents which position has already been occupied (See Fig.1). The effectiveness of this strategy was initially explored in simulations. Further, the recently published multiple pheromone communication platform named ColCOS $\Phi$ [15] provides us with a good opportunity to extend and deepen the swarm robotic studies inspired by multiple pheromones from simulations [10], [11] to real robots application. The main performances and results from simulations are in line with that of real robot experiments conducted in $\operatorname{ColCOS} \Phi$, demonstrating the effectiveness of this deployment strategy. Aligned simulation and real robot experiment results demonstrate the significant role each pheromone plays in completing the deployment task. In addition, we further explore this strategy by parameter sensitive testing which is helpful and inspirational for strategy design of swarm intelligence based on similar ideas.

To conclude, salient contributions of this study can be summarised as; (a) a novel attempt to involve multiple pheromones in solving collective task not only in simulation but also real robots, filling the gap that many studies just consider one pheromone at a time, (b) systematic investigation of the simple deployment strategy in simulation and physical world provides suggestions for related strategy design, (c) implementing the concept of multiple pheromones from social insects to swarm robotics that may help biologists in 
real insects research, and (d) a new application of ColCOS $\Phi$ and demonstrate its effectiveness and feasibility for swarm robotics study.

The other sections completing this paper are organized as follows: II gives the methods and model of this deployment strategy; III introduces the specific experiments settings for the simulation and real robots; IV displays and analyses the experimental results; $\mathrm{V}$ gives the conclusion and the future work.
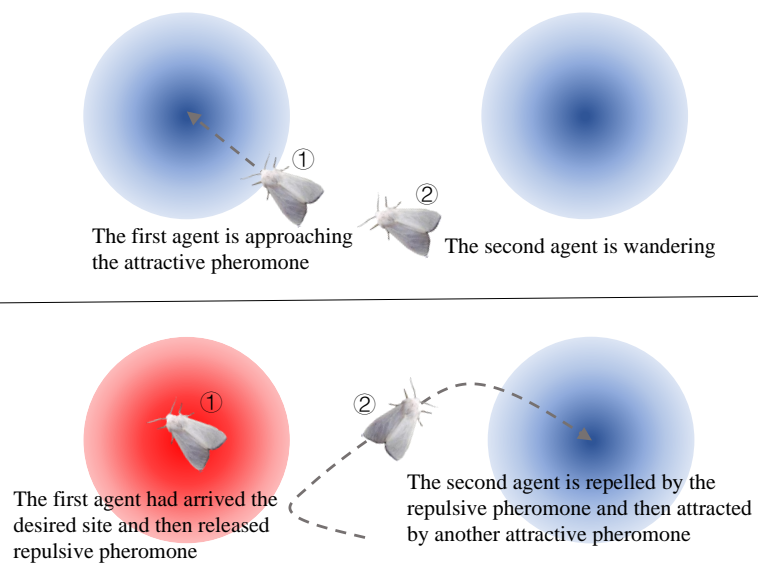

Fig. 1. The schematic diagram of the deployment strategy.

\section{Materials AND Methods}

The specific deployment task in this paper is illustrated in Figure 2. The agents we employ do not have any prior knowledge of the global map and the ability to communicate with each other. In order to deploy such simple swarm robots from the initial arbitrary distribution to the desired distribution, a multiple pheromone based strategy is designed that is inspired by the insects' reaction to different kinds of pheromones (Fig.1). In this strategy, every single position partly forming the goal pattern is marked by the attractive pheromone. Once an agent arrives at one of those single positions, it will secret the repellent pheromone at that position to prevent other agents from twice occupying the spot.

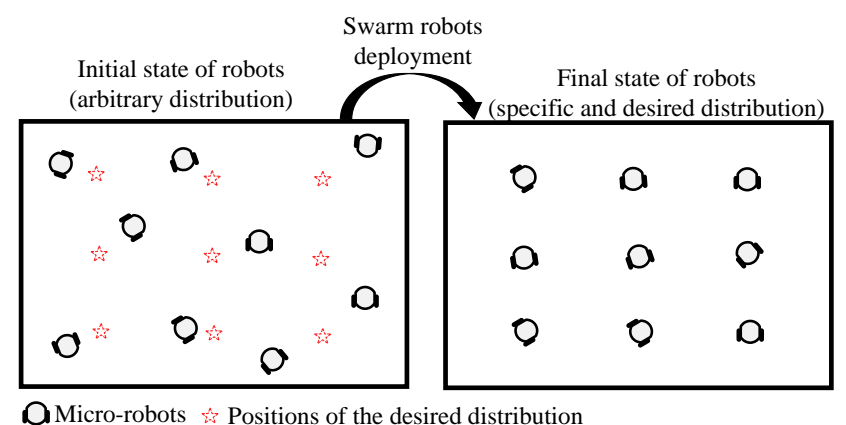

Fig. 2. The schematic diagram of the deployment task we want to complete.

\section{A. Multiple Pheromone Model}

Pheromone is a kind of volatile compound whose concentration changes with respect to time that can be modelled by a differential equation with evaporation and diffusion parameters [16]. To simplify the calculation and make it more suitable for our strategy, the 2D spatial distribution of pheromone is modelled by a scaled bivariate normal distribution as shown in (1) with the assumption that the pheromone will keep emitting from the source.

$$
\begin{aligned}
P(x, y)= & \frac{K}{2 \pi \sigma_{x} \sigma_{y} \sqrt{1-\rho^{2}}} \cdot \\
& e^{-\frac{1}{2\left(1-\rho^{2}\right)}\left[\frac{\left(x-\mu_{x}\right)^{2}}{\sigma_{x}^{2}}+\frac{\left(y-\mu_{y}\right)^{2}}{\sigma_{y}^{2}}-\frac{2 \rho\left(x-\mu_{x}\right)\left(y-\mu_{y}\right)}{\sigma_{x} \sigma_{y}}\right]}
\end{aligned}
$$

In (1), $\left(\mu_{x}, \mu_{y}\right)$ defines the source position of the pheromone, $K$ is the scale factor defining the strength of the pheromone, $\sigma_{x}$ and $\sigma_{y}$ are the standard deviation of Gaussian function which in this context determine the diffusion effect of pheromone. Given the natural condition where there is no wind and other disturbances, the covariance $\rho$ should be zero and $\sigma_{x}=\sigma_{y}=\sigma$, which means that the pheromone is spreading equally in $x$ and $y$ direction in the Cartesian coordinates. Therefore, (1) is simplified as,

$$
P(x, y)=\frac{K}{2 \pi \sigma^{2}} e^{-\frac{1}{2}\left[\frac{\left(x-\mu_{x}\right)^{2}}{\sigma^{2}}+\frac{\left(y-\mu_{y}\right)^{2}}{\sigma^{2}}\right]}
$$

In a $2 \mathrm{D}$ environment where the robot will be deployed, the strength of the pheromone with $i^{\text {th }}$ type at position $(x, y)$ is the linear summation of all the presented $k^{\text {th }}$ pheromone where $k=1,2 \ldots n$, which is formulated in (3).

$$
P_{i}(x, y)=\sum_{k=1}^{n} P_{i, k}(x, y)
$$

\section{B. Behaviour Control}

In our deployment strategy, pheromone is the only cue that the agent can sense to complete the deployment task except for some necessary sensory information to avoid collision and obstacles. Given these limited sensory inputs, a suitable behaviour control strategy should be designed to guide the agent to navigate in the bordered 2D world and finally finish the task.

1) Finite State Machine: A finite state machine (FSM) for controlling the agents' behaviour to eventually achieve the goal of deployment should meet the following three principles,

- The agent can navigate to the desired location and stop on it to complete its own task. Here the attractive pheromone will be useful.

- The agent should not navigate to the location that has already been occupied by another agent. Here we can make good use of the repellent pheromone.

- The agent can explore the world with the ability to avoid collisions, which provides the agent with possibilities to sense the pheromone. Because the defined attractive 
pheromone cannot diffuse so fast that the whole deployment area is covered.

Based on the aforementioned principles, a compact FSM is designed and illustrated in Figure 3. Once the agent starts its deployment task and finishes the initialisation, it first goes to the wandering mode, in which the agent will freely explore the environment. Once the agent arrives at the diffusion range of the pheromones, the pheromone guide motion mode will be triggered. If eventually the sensed value of the attractive pheromone is greater than the threshold, the agent will stop. Necessarily, to safely and continuously explore the environment, once the agent encounters an obstacle (other agents or the boundary of the world), the avoiding collision mode will override the controller to avoid the looming collision.

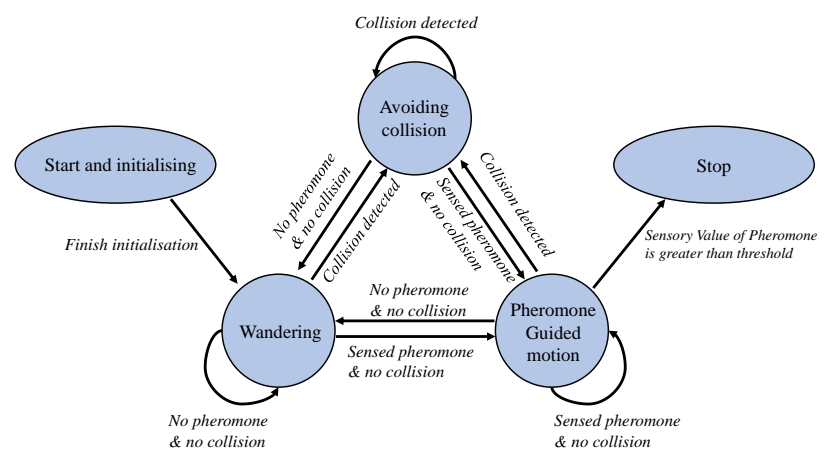

Fig. 3. The finite state machine of the agent in deployment.

2) Motion Control Model: The implementation details of the motion control mode for every state in the FSM are introduced as,

- Start and initialising: Do the necessary preparation, such as initialise peripherals and parameters.

- Wandering: Go forward with a constant basic speed $\left(V_{0}\right)$ and a random turn $\left(\theta_{w}\right)$.

- Pheromone guided motion: To guide the agent to the centre of the attractive pheromone, a bio-inspired gradient tracking method is designed [17]. The attractive and repellent pheromones' effect on the motion can be represented as two vectors formulated in (4), where $P_{a}$ and $P_{r}$ defines the strength of the sensed attractive and repellent pheromone, respectively. $\theta_{a}$ and $\theta_{r}$ stand for the positive gradient directions. Note that for the attractive pheromone, the desired motion direction is in line with the positive gradient direction while for the repellent pheromone, these two directions are opposite. To integrate the guidances from these two kinds of pheromones into one motion decision, a bio-inspired method is applied to do the optimal integration of circular cues [18], [19]. Hence the integrated motion direction $(\theta)$ is obtained by vector summation $\vec{M}=$ $\vec{M}_{a}+\vec{M}_{r}=(M, \theta)$ and $\theta$ is calculated by (5).

- Avoiding collision: Make a sharp turn $\left(\theta_{a}\right)$ away from the obstacle.

$$
\vec{M}_{a}=\left(P_{a}, \theta_{a}\right), \vec{M}_{r}=\left(P_{r}, \theta_{r}+\pi\right)
$$

$$
\theta=\arctan \frac{P_{a} \sin \theta_{a}+P_{r} \sin \left(\theta_{r}+\pi\right)}{P_{a} \cos \theta_{a}+P_{r} \cos \left(\theta_{r}+\pi\right)}
$$

\section{EXPERIMENTAL SETUP}

To systematical investigate the efficiency of multiple pheromones as compared to single pheromone applications, we explore this multiple pheromones based deployment both in the simulation context and the real robotic scenario. Experiments in simulation and real robot both start with $N$ robots located in random positions with random headings, where the $N$ desired positions are marked by the attractive pheromones. All the individual agents will simultaneously finish the initialisation step and shift to the wandering state (Fig.3). The successful deployment will be verified if all the agents have stopped and released the repellent pheromone at the desired positions before exceeding the defined experimental time $T_{\text {out }}$.

\section{A. Simulations}

The deployment strategy is implemented by Python and executed in the Jupyter iPython. The environment is composed of the following sections.

1) Arena Setup: We define a simulated rectangular arena with $0.8 \mathrm{~m}$ width and $1.43 \mathrm{~m}$ length (similar to the size of our real robot arena), within which the simulated pheromones are released and the simulated robots must begin exploring. An example of the pheromone implementation is shown by a heat map in the left part of Figure 4, where the darker colour represents a higher concentration of pheromone.

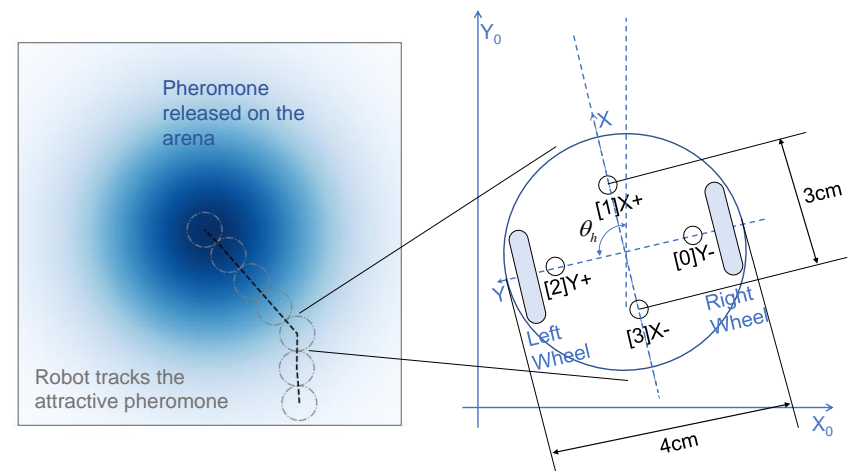

Fig. 4. The implementation of pheromones and robots in the simulated environment. Sensors of the agent are labelled with $j \in\{0,1,2,3\}$ corresponding to $Y+, X+, Y-, X-$ in this top view figure. The world's and robot's coordinates are labelled with $X_{0}, Y_{0}$ and $X, Y$, respectively.

2) Simulated Robot: The robot is simulated as a $4 \mathrm{~cm}$ diameter circle depicted in Figure 4. Four colour sensors are simulated as four points distributed in a $2 \times 2$ array with $3 \mathrm{~cm}$ diagonal distance to efficiently and conveniently get the instantaneous gradient of the pheromone. The concentration of the pheromone sensed by each sensor is calculated by (3)(2) and the average value is regarded as the current pheromone concentration sensed by the robot. The positive gradient direction of the pheromone in the robot's coordinates can be computed by (6), where $i \in\{1,2\}$ indicates two channels 


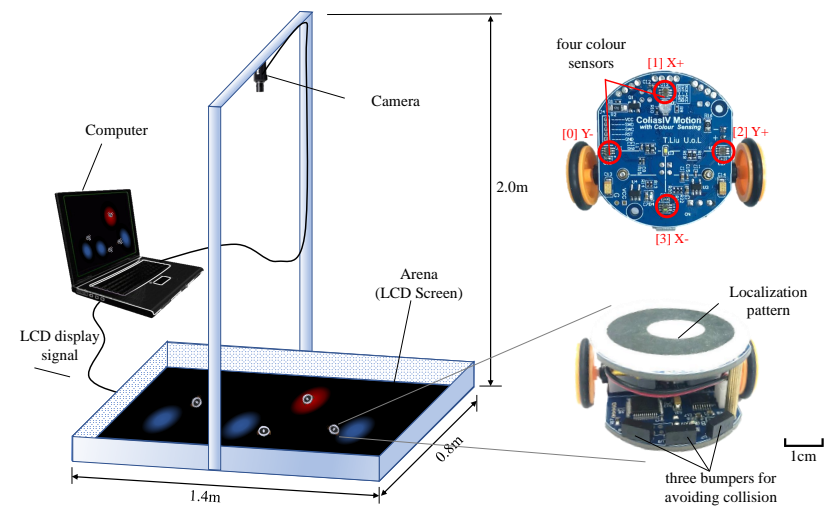

Fig. 5. The structure of $\operatorname{ColCOS} \Phi$ and the robotics platform. Key components are labelled, and sensors of a robot mounted as the same with the simulation agent are shown in the upper right corner with a bottom view.

of the simulated sensor to sense the attractive and repellent pheromone. Then the direction $\theta_{i(X, Y)}$ in robot's coordinates can be transformed to the world coordinates $(\mathrm{X} 0, \mathrm{Y} 0)$ by (7), where $\theta_{h}$ is the heading direction of the agent in the world coordinates. In sequence, the desired motion direction driven by pheromone can be calculated by (5). In the simulation context, we have ideal motion control, so we just force the agent's motion direction to be $\theta$, and the speed of the simulated robot is set to be a constant $v=4 \mathrm{~cm} / \mathrm{s}$.

$$
\begin{gathered}
\theta_{i(X, Y)}=\arctan \frac{P_{i, 2}-P_{i, 0}}{P_{i, 1}-P_{i, 3}} \\
\theta_{i\left(X_{0}, Y_{0}\right)}=\theta_{i(x, y)}+\theta_{h}
\end{gathered}
$$

\section{B. Real Robot Experiments}

The real robot experiments implement a platform called $\operatorname{ColCOS} \Phi$ [15]. It is a specially designed platform for multiple pheromones research. This novel platform uses different colours to encode pheromones. In this part, we briefly introduce the platform and robots control logic. Then, a simple test was shown to illustrate the efficiency of this platform.

1) Arena Setup: The arena consists of four parts (a camera, a screen, a PC and a frame) as shown in the left part of the Figure 5.

Based on the hardware mentioned above, two main algorithms are executed on the computer; (a) the localisation algorithm [20] based on pattern cognition is applied to track the robots' position, and (b) the algorithm to display the virtual pheromones on the screen. The same with the simulation, the pheromone implementation also based on (2). Different from simulation context, the pheromone is implemented theoretically while for the real robot experiment, according to $\operatorname{ColCOS} \Phi$, the strength of the virtual pheromone in the physical world is implemented by the RGB value of the pixel. The pheromone type $i$ is realised by the colour of that pixel. In this study, we set the attractive and repellent pheromone to be blue and red respectively then, the RGB value of a pixel $I(x, y)=(r, g, b)$ can be calculated by $(8)$.

$$
\left\{\begin{array}{l}
r=\sum_{k=1}^{n} P_{a, k}(x, y) \\
g=0 \\
b=\sum_{k=1}^{n} P_{r, k}(x, y)
\end{array}\right.
$$

The RGB value of a pixel $I(x, y)=(r, g, b)$ at the $x^{\text {th }}$ row and $y^{\text {th }}$ column of the screen can represent three kinds of pheromones with the normalised strength from $0-255$. Where the $P_{a}$ and $P_{r}$ define the strength of the attractive and repellent pheromone, respectively. $k$ stands for the number of the similar type pheromones.

2) The Micro Robot Description: The right part of Figure 5 illustrates the robotic agent. To comply with the ColCOS $\Phi$ system accurately, four colour sensors are mounted on the robot in the same way as the simulated robot to get the necessary value for gradient calculation. These sensors measure the intensity of brightness, red, green and blue component of the ambient light by four independent 16-bit ADC channels. However, in this study, only the blue and red channels are utilised. On top of the robot, a special pattern is attached for the localisation system to recognise the robot and track its position. Three bumpers are installed in front of the robots to dampen collisions.

3) Robot Motion Control: Similar with the simulated robots, the concentration of pheromone can be calculated by (3), and the average readout value of four sensors can be taken as $P_{a}$ and $P_{r}$ which is the current pheromone concentration sensed by the robot. The positive gradient direction $\theta_{a}$ and $\theta_{r}$ can be calculated by (6). Then, the desired direction $\theta$ can be calculated through (4) and (5).

Unlike the ideal control mechanism of the simulated robot, the real robot motion is driven by two wheels. Therefore, the desired motion direction should be transformed into left and right motor rotational speeds $\left(R_{l}\right.$ and $\left.R_{r}\right)$ that are calculated by (9) and (10), respectively as,

$$
\begin{aligned}
& R_{l}=v_{b}-G_{t} \theta \\
& R_{r}=v_{b}+G_{t} \theta
\end{aligned}
$$

Where $v_{b}$ stands for the basic forward speed, generally defined as $4-5 \mathrm{~cm} / \mathrm{s}$. And $G_{t}$ is the parameter for adjusting the responding speed of turning.

4) The Preliminary Test: In order to verify whether the ColCOS $\Phi$ system meets the scope of this study, we initially undertake a preliminary test illustrated in Figure 6(a). Three spots of artificial pheromone are displayed on the LCD screen. The robot follows the depicted grey trajectory to pass through the surface of the attractive pheromone freely, and then it reacts to the pheromone with the controller enabled, resulting in the white trajectory shown in Figure 6(a). During this whole process, the sensory value has been collected and then plotted in Figure 6(b,c,d), from which we can see the correct Gaussian distribution of the pheromone concentration, the expected motion of the robot and the immediate pheromone releasing. 

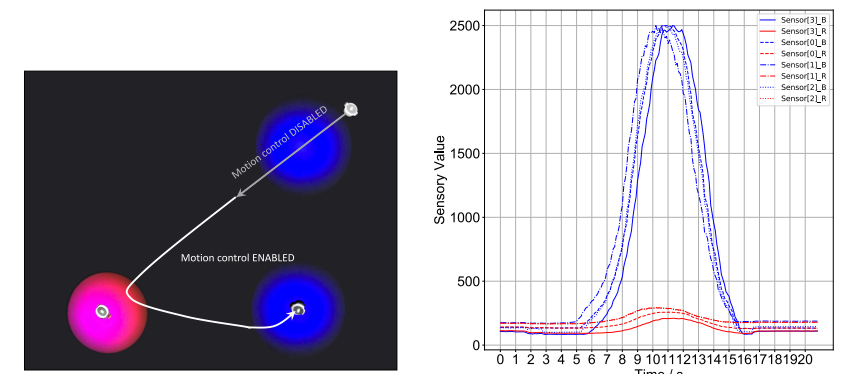

(a) Trajectory of the robot to collect sensory data

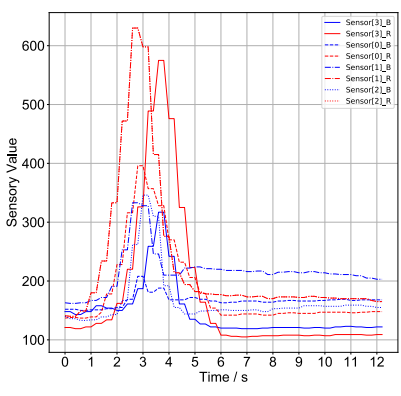

c) Sensory value when repelled by repelllent pheromone

Fig. 6. The preliminary test of the real robot experimental system.

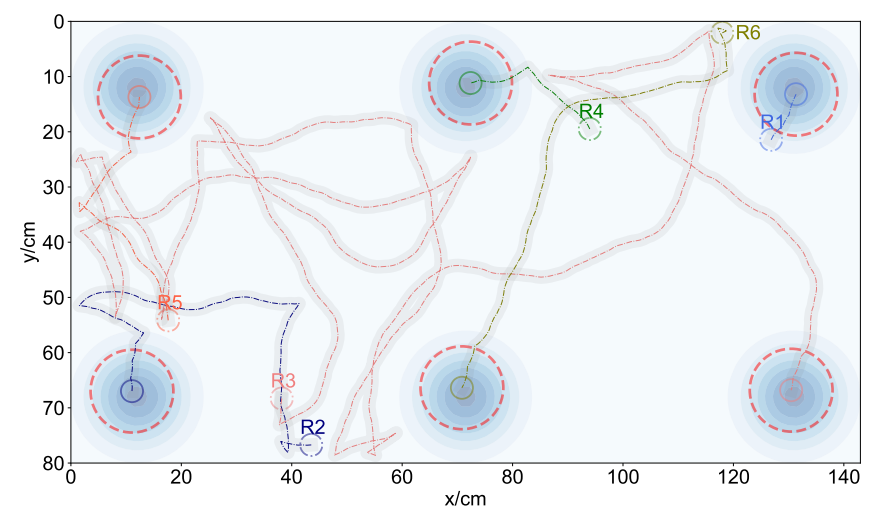

Fig. 7. The simulation results of the typical performance of our deployment strategy model. Trajectories and headings of six robots are depicted with colourful quivers, red dash circles indicate the half-width of the Gaussian modelled repellent pheromone.

\section{RESUlts AND Discussion}

Both simulations and real robot experiments are demonstrated to systematically investigate the roles that multiple pheromones play in solving the deployment tasks introduced in Figure 2. In addition, we also explore how the parameters of multiple pheromones affect the deployment performances.

\section{A. General Deployment Performance}

The typical performance of our deployment strategy is illustrated in Figures 7 and 8 for the simulations and the real robot experiments, respectively. In these experiments, it is evident how a simple strategy delivers an effective deployment performance. Six robots with slow speed (around $4 \mathrm{~cm} / \mathrm{s}$ ) are uniformly distributed within the arena, that is 250 times its size for one minute.

After validating the effectiveness of the designed deployment strategy, next we make an attempt to test if the task can
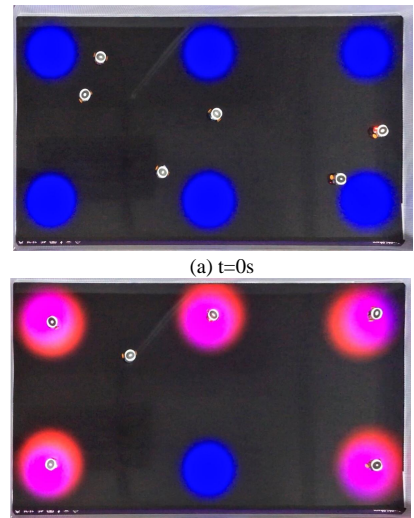

(c) $\mathrm{t}=45 \mathrm{~s}$

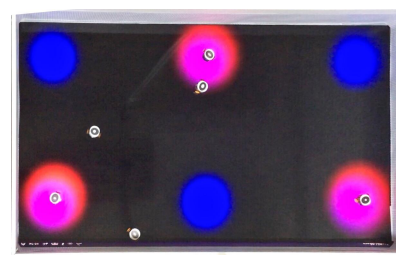

(b) $t=25 \mathrm{~s}$

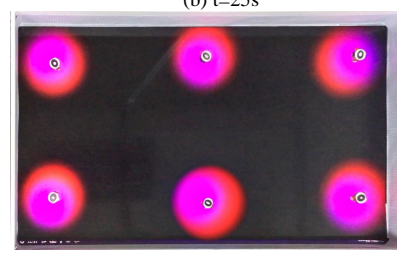

(d) $\mathrm{t}=59 \mathrm{~s}$
Fig. 8. The real robot results of the typical performance of our deployment strategy model. The time stamp is shown in the captions of sub-figures.

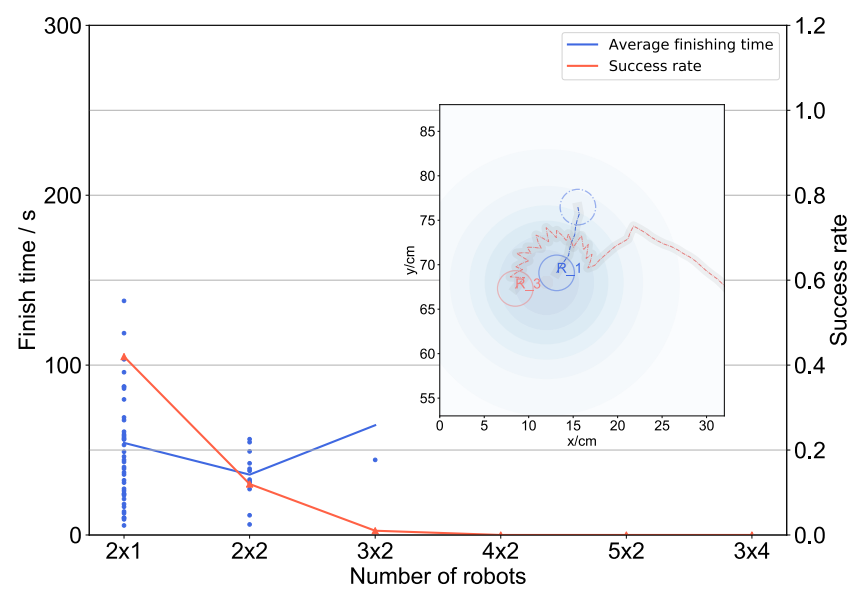

Fig. 9. The simulation results of the deployment performance involving single pheromone. Inserted figure shows a typical failure caused by elimination of the repellent pheromone.

still be completed in the absence of the repellent pheromone.

\section{B. The Function of Multiple Pheromone}

In order to identify the function of multiple pheromones, simulation of single pheromone (only the attractive pheromone) based deployment is conducted and the results are shown in Figure 9. Two criteria are applied to assess the performance; the time consumed for finishing the deployment (finishing time $T f$ ), and the success rate $(\mathrm{Sr})$ that is the ratio of expected deployment (all finally arrived robots at the desired positions) to the number of trials. It is obvious that in the absence of repellent pheromone, the deployment task is very difficult to be achieved, which is implied by the extremely low value of $S r$. The inserted figure of Figure 9 clearly shows why the deployment fails. If there is no repellent pheromone, a desired position occupied by an agent still attracts other agents. Thus there will be a high possibility of more than one agent occupying the same spot.

After analyzing the effects of multiple pheromones and single pheromone, we empirically take two factors into account which may have a crucial effect on the performance 
to do the further analysis; (a) the number of robots (size of the swarm), and (b) diffusion of the pheromones.

\section{Size of the Swarm}

To investigate the influence of the number of robots on the deployment performance, deployment tasks with different number of robots have been performed in both the simulated environment as well as the real-world arena.

1) Simulation Results: In this test, we fixed the diffusion of the attractive and repellent pheromones, i.e., mathematically we fixed the values for $\sigma_{x}$ and $\sigma_{y}$ in (2) of the two pheromones to be $6 \mathrm{~cm}$ and $7.5 \mathrm{~cm}$. The desired locations are arranged in a rectangle array with different sizes from $2 \times 1$ to $4 \times 3$, corresponding to the number of robots $N$ ranging from 2 to 12 with an increment of 2 , running each group for 100 trials. Simulation results of the varied number of robots are shown in Figure 10(a), from which we can see that $\mathrm{Sr}$ decreases slowly with the number of robots increased, but when the robots exceed $5 \times 2=10, S r$ decreases dramatically. The reason behind this phenomenon is due to the space constraints (arena size), that is when the number of robots increase beyond a certain threshold, the arena will be so crowded that the robots get blocked and confined into a small space illustrated in Figure 10(e). There is a very little chance for the robot to pass through from one side to another reaching its destination (see $R 7$ fails to pass through in Figure 10(e)). The average time consumed linearly and slightly increases with $N$. From the distribution of the time used (blue dots in Figure 10(a)), we can find a large variance caused by the random initial positions and headings of robots, but most of the dots drop below the average value which also suggests the efficiency of this deployment strategy.

2) Real Robot Results: Due to the hardware and time constraints, we test the effect of agent population (number of robots) on the deployment performance with three groups: $2,2 \times 2$ and $3 \times 2$, and for each group, we run 20 trials maintaining other parameters such as the diffusion of the pheromones same as in the simulations. Experimental results are shown in Figure 10(b), it is evident that the whole trend is in agreement with the simulation results, i.e. $T f$ slightly increases and success rate $S r$ drop as the number of robots increase.

\section{Diffusion of the Pheromones}

Diffusion of the pheromones can be modelled by the variance of the Gaussian distribution, namely, $\sigma$ in (2). High variance implies fast diffusion, thus the pheromones' effect shall propagate to a wider area that in turn attract or repel agents farther away, and inversely lower variance represents pheromones with slower diffusion.

1) Simulation Results: In this simulation test, we set $\sigma_{A}$ of attractive pheromones to range from $5 \mathrm{~cm}$ to $8 \mathrm{~cm}$ with steps of $1 \mathrm{~cm}$, and the diffusion factor of repellent pheromone $\sigma_{R}=\sigma_{A}+1.5$. The number of robots is fixed to 6 , so the attractive pheromones are placed on a $3 \times 2$ array. Again for each group, we run 100 trials. Results are shown in Figure 10 (c), we can see that the $S r$ decays with the increase in the radius of pheromone and will significantly decrease when the radius exceeds $7 \mathrm{~cm}$, although the $T f$ slightly drops as the diffusion gains momentum.

2) Real Robot Results: To verify how the diffusion of the pheromone influences the deployment performance of real robots, we performed the same tests as in the simulations except for the number of trials for each group was changed from 100 to 20. Results are illustrated in Figure 10(d), the whole trend of the curve is consistent with the simulation, implying that faster diffusion costs lesser time for the deployment to complete but at a possibly lower success rate. One such experiment is shown in Figure 10(f) to illustrate why fast diffusion will cause deployment failure. The reason is that the possibility of a position being occupied by more than one agent will raise with an increase in diffusion factor.

Considering all the above results, it can be inferred that the population density of the robots actually impacts the performance, because the arena size is fixed throughout the simulation and real robot experiments. Therefore, while distributing swarm robots in a certain area using this strategy, the number of robots $N$ and the diffusion factor of pheromone $\sigma$ should be carefully selected to obtain an efficient deployment performance at high success rate.

\section{CONCLUSIONS AND FUTURE WORK}

The concept of multiple pheromones derived from insect studies [12] has been realised in swarm intelligence through a compact deployment strategy. By investigating the performances and properties of this strategy not only in simulation but in a physical system $(\operatorname{ColCOS} \Phi)$, the function of individual pheromone has been verified. These results lead to the conclusion that multiple pheromones' incorporation can improve the efficiency of a collective task, which is in agreement with the related literature. Through a systematic analysis of parameter-sensitive testing of size of the swarm, and diffusion of the pheromone, we concluded that both factors have a significant effect on the deployment performance.

For future work, on one hand, more types of pheromones will be taken into account to investigate more complex collective behaviours and test whether efficiency can be further improved. On the other hand, exploring other challenging problems in swarm intelligence that can be potentially solved by multiple pheromones is highly recommended.

\section{REFERENCES}

[1] P. Karlson and M. Lüscher, "'Pheromones': A new term for a class of biologically active substances," Nature, 1959.

[2] M. S. Blum and J. M. Brand, "Social insect pheromones: Their chemistry and function," Integrative and Comparative Biology, 1972.

[3] Z. RÚŽIČKA, "Oviposition-deterring pheromone in chrysopa oculata (neuroptera: Chrysopidae)," Eur. J. Entomol, vol. 91, pp. 361-370, 1994.

[4] L. Bayindir, "A review of swarm robotics tasks," Neurocomputing, vol. 172, pp. 292-321, 2016.

[5] S. Berman, A. Halász, V. Kumar, and S. Pratt, "Bio-inspired group behaviors for the deployment of a swarm of robots to multiple destinations," in Proceedings 2007 IEEE International Conference on Robotics and Automation. IEEE, 2007, pp. 2318-2323. 

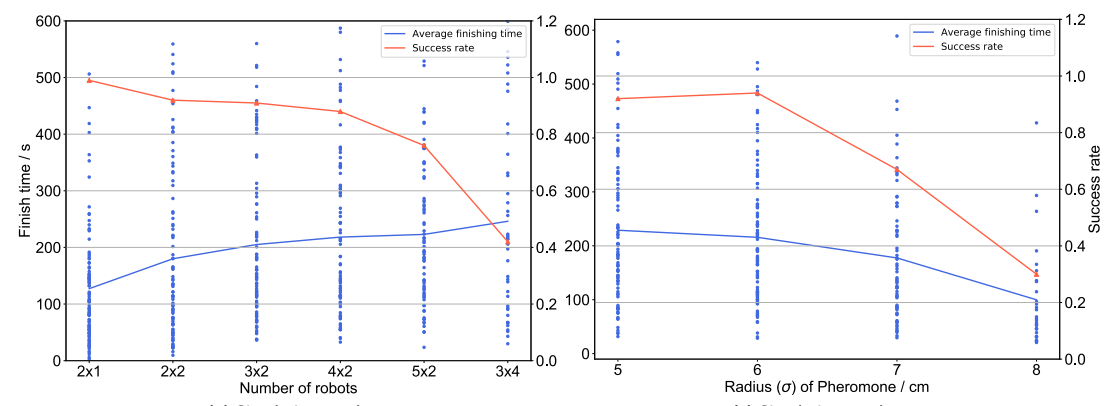

(a) Simulation results (c) Simulation results
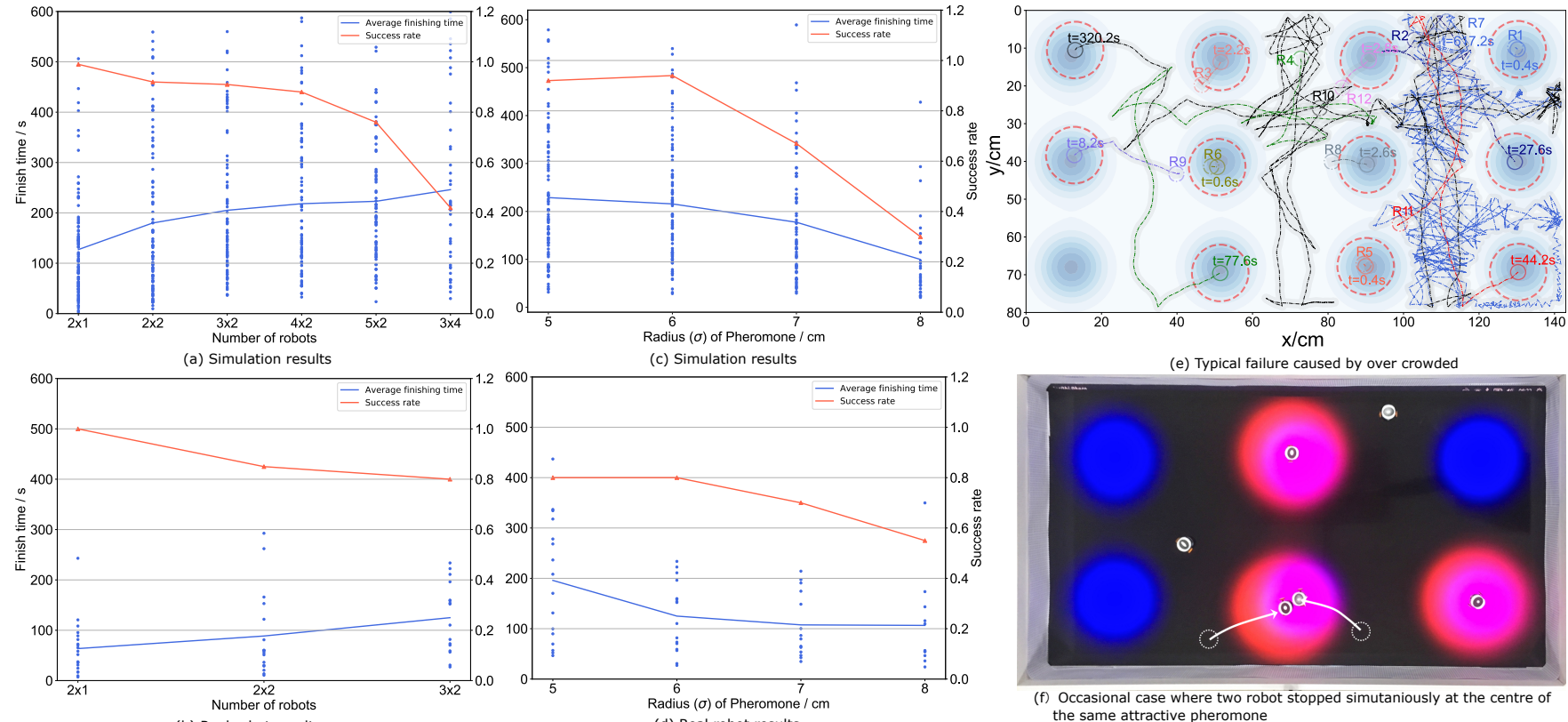

(b) Real robot results (d) Real robot results

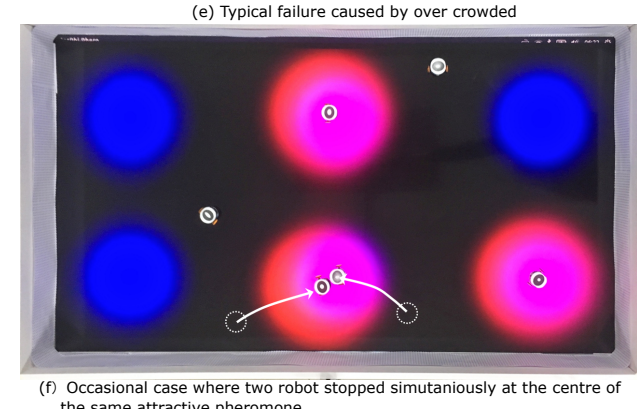

the same attractive pheromone

Fig. 10. The results of parameter sensitivity test for simulation and real robot experiments, and special case for explaining the failure of the deployment.

[6] S. Kernbach, R. Thenius, O. Kernbach, and T. Schmickl, "Reembodiment of honeybee aggregation behavior in an artificial microrobotic system," Adaptive Behavior, vol. 17, no. 3, pp. 237-259, 2009.

[7] D. Payton, M. Daily, R. Estowski, M. Howard, and C. Lee, "Pheromone robotics," Autonomous Robots, vol. 11, no. 3, pp. 319324, 2001.

[8] I. Caliskanelli, B. Broecker, and K. Tuyls, "Multi-robot coverage: A bee pheromone signalling approach," in Artificial Life and Intelligent Agents Symposium. Springer, 2014, pp. 124-140.

[9] F. Aznar, M. Pujol, R. Rizo, F. Pujol, and C. Rizo, "Energy-efficient swarm behavior for indoor uav ad-hoc network deployment," Symmetry, vol. 10, no. 11, p. 632, 2018.

[10] J. A. Sauter, R. Matthews, H. Van Dyke Parunak, and S. A. Brueckner, "Performance of digital pheromones for swarming vehicle control," in Proceedings of the fourth international joint conference on $A u-$ tonomous agents and multiagent systems. ACM, 2005, pp. 903-910.

[11] G. Silva, J. Costa, T. Magalhães, and L. P. Reis, "Cyberrescue: A pheromone approach to multi-agent rescue simulations," in 5th Iberian Conference on Information Systems and Technologies. IEEE, 2010, pp. $1-6$.

[12] E. J. H. Robinson, D. E. Jackson, M. Holcombe, and F. L. W. Ratnieks, "Insect communication: No entry' signal in ant foraging," Nature, vol. 438, no. 7067, pp. 442-442, 2005. [Online]. Available: http://www.nature.com/doifinder/10.1038/438442a

[13] A. Dussutour, S. C. Nicolis, G. Shephard, M. Beekman, and D. J. T. Sumpter, "The role of multiple pheromones in

[17] G. M. Barbara and J. G. Mitchell, "Bacterial tracking of motile algae," FEMS microbiology ecology, vol. 44, no. 1, pp. 79-87, 2003. food recruitment by ants," Journal of Experimental Biology, vol. 212, no. 15, pp. 2337-2348, 2009. [Online]. Available: http://jeb.biologists.org/cgi/doi/10.1242/jeb.029827

[14] A.-J. Garcia-Sanchez, F. Garcia-Sanchez, and J. Garcia-Haro, "Wireless sensor network deployment for integrating video-surveillance and data-monitoring in precision agriculture over distributed crops," Computers and electronics in agriculture, vol. 75, no. 2, pp. 288-303, 2011.

[15] X. Sun, T. Liu, C. Hu, Q. Fu, and S. Yue, "ColCOS $\Phi$ : A multiple pheromone communication system for swarm robotics and social insects research," in 2019 4th IEEE International Conference on Advanced Robotics and Mechatronics, ICARM 2019, 2019.

[16] F. Arvin, T. Krajnik, A. E. Turgut, and S. Yue, "COS $\Phi$ : Artificial pheromone system for robotic swarms research," in IEEE International Conference on Intelligent Robots and Systems, vol. 2015-December, 2015, pp. 407-412.

[18] X. Sun, M. Mangan, and S. Yue, "An analysis of a ring attractor model for cue integration," in Conference on Biomimetic and Biohybrid Systems. Springer, 2018, pp. 459-470.

[19] T. Hoinville and R. Wehner, "Optimal multiguidance integration in insect navigation," Proceedings of the National Academy of Sciences, vol. 115, no. 11, p. 201721668, 2018. [Online]. Available: http://www.pnas.org/lookup/doi/10.1073/pnas.1721668115

[20] T. Krajník, M. Nitsche, J. Faigl, P. Vaněk, M. Saska, L. Přeučil, T. Duckett, and M. Mejail, "A practical multirobot localization system," Journal of Intelligent \& Robotic Systems, vol. 76, no. 3-4, pp. 539-562, 2014 